\title{
Random forest-based modelling to detect biomarkers for prostate cancer progression
}

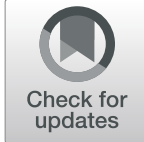

Reka Toth ${ }^{1 \dagger}$, Heiko Schiffmann ${ }^{2 \dagger}$, Claudia Hube-Magg ${ }^{2}$, Franziska Büscheck ${ }^{2}$, Doris Höflmayer ${ }^{2}$, Sören Weidemann², Patrick Lebok ${ }^{2}$, Christoph Fraune ${ }^{2}$, Sarah Minner ${ }^{2}$, Thorsten Schlomm²,3 Guido Sauter ${ }^{2}$, Christoph Plass ${ }^{1,4}$, Yassen Assenov ${ }^{1}$, Ronald Simon ${ }^{2+}$, Jan Meiners ${ }^{5+}$ and Clarissa Gerhäuser ${ }^{1 *+}$ (D)

\begin{abstract}
Background: The clinical course of prostate cancer (PCa) is highly variable, demanding an individualized approach to therapy. Overtreatment of indolent PCa cases, which likely do not progress to aggressive stages, may be associated with severe side effects and considerable costs. These could be avoided by utilizing robust prognostic markers to guide treatment decisions.

Results: We present a random forest-based classification model to predict aggressive behaviour of prostate cancer. DNA methylation changes between PCa cases with good or poor prognosis (discovery cohort with $n=70$ ) were used as input. DNA was extracted from formalin-fixed tumour tissue, and genome-wide DNA methylation differences between both groups were assessed using Illumina HumanMethylation450 arrays. For the random forest-based modelling, the discovery cohort was randomly split into a training (80\%) and a test set (20\%). Our methylation-based classifier demonstrated excellent performance in discriminating prognosis subgroups in the test set (Kaplan-Meier survival analyses with log-rank $p$ value $<0.0001$ ). The area under the receiver operating characteristic curve (AUC) for the sensitivity analysis was 95\%. Using the ICGC cohort of early- and late-onset prostate cancer $(n=222)$ and the TCGA PRAD cohort $(n=477)$ for external validation, AUCs for sensitivity analyses were $77.1 \%$ and $68.7 \%$, respectively. Cancer progression-related DNA hypomethylation was frequently located in 'partially methylated domains' (PMDs) - large-scale genomic areas with progressive loss of DNA methylation linked to mitotic cell division. We selected several candidate genes with differential methylation in gene promoter regions for additional validation at the protein expression level by immunohistochemistry in $>12,000$ tissue micro-arrayed PCa cases. Loss of ZIC2 protein expression was associated with poor prognosis and correlated with significantly shorter time to biochemical recurrence. The prognostic value of ZIC2 proved to be independent from established clinicopathological variables including Gleason grade, tumour stage, nodal stage and prostate-specific-antigen.

Conclusions: Our results highlight the prognostic relevance of methylation loss in PMD regions, as well as of several candidate genes not previously associated with PCa progression. Our robust and externally validated PCa classification model either directly or via protein expression analyses of the identified top-ranked candidate genes will support the clinical management of prostate cancer.
\end{abstract}

\footnotetext{
* Correspondence: c.gerhauser@dkfz.de

${ }^{\dagger}$ Reka Toth, Heiko Schiffmann and Ronald Simon contributed equally to this

work.

†Jan Meiners and Clarissa Gerhäuser share co-senior authorship.

${ }^{1}$ Cancer Epigenomics, German Cancer Research Center (DKFZ), 69120

Heidelberg, Germany

Full list of author information is available at the end of the article
}

(c) The Author(s). 2019 Open Access This article is distributed under the terms of the Creative Commons Attribution 4.0 International License (http://creativecommons.org/licenses/by/4.0/), which permits unrestricted use, distribution, and reproduction in any medium, provided you give appropriate credit to the original author(s) and the source, provide a link to the Creative Commons license, and indicate if changes were made. The Creative Commons Public Domain Dedication waiver (http://creativecommons.org/publicdomain/zero/1.0/) applies to the data made available in this article, unless otherwise stated. 


\section{Background}

Prostate cancer $(\mathrm{PCa})$ is the second most prevailing cancer in the male population worldwide, an estimated 1.28 Mio. newly diagnosed cases and 350,000 cancer-related deaths in 2018 [1]. Although the aetiology of prostate cancer is controversial, it is likely to result from accumulating DNA damage in stress-exposed ageing prostate epithelial cells [2]. Specifically, chromosomal rearrangements and oncogene fusion genes in these cells are driven by androgens [3, 4]. Despite a large number of studies that have suggested a multitude of candidate prognostic markers in prostate cancer, none of these genes has proven to be superior over the established histological prognostic factors including tumour stage and Gleason grade. Localized prostate cancer with low Gleason score usually remains indolent, requiring only active surveillance or minimal treatment. Nevertheless, many patients may be over-treated with associated side effects and substantial costs [5]. There is, therefore, general agreement that novel specific biomarkers for the diagnosis and prognosis of prostate cancer are needed for an efficient clinical management of this disease [6,7].

Recent high-resolution genome-wide studies have significantly improved our understanding of chromosomal and genetic alterations associated with prostate cancer development, such as the androgen-driven formation of gene fusions between the transmembrane serine protease TMPRSS2 and a member of the oncogenic ETS transcription factor family like ERG in about $50 \%$ of all prostate cancer cases, and frequent loss of the tumour suppressor gene PTEN $[4,8,9]$. These events affect signalling pathways and lead to alterations in gene expression programs that have been used for the development of gene signatures (genomic classifiers) as biomarkers for the prediction of prostate cancer prognosis [10]. Although several studies have demonstrated some prognostic value of gene expression-based signatures, due to the limited stability of RNA and often low quality when extracted from formalin-fixed paraffin-embedded (FFPE) material, protein- or DNA-based methods might be superior to RNA expression profiles for biomarker development.

There is substantial evidence that genetic defects in prostate cancer are complemented or even preceded by epigenetic aberrations such as DNA methylation [11]. Novel technologies based on genome-wide screens for aberrant DNA methylation and epigenetic gene silencing, including the widely used Illumina 450k Beadchip arrays, have allowed identification of hundreds of genes aberrantly methylated during prostate cancer development $[3,8,11$, 12]. These cancer-specific epigenetic alterations have been shown to enable the development of methylation-based assays to distinguish between benign and malignant tissue and to predict the course of the disease $[11,13,14]$.
In recent years, machine-learning techniques became widely used in modern molecular research to build predictive models [15]. Random forest [16] is an ensemble learning method based on the construction of many classification trees. Main benefits of the method are its robustness against overfitting, user-friendliness and the easy interpretation of the model [16].

Our goal was to use random forest-based modelling of DNA methylation alterations to develop a classifier predicting the outcome of prostate cancer. In addition, the tight connection of DNA methylation events with gene expression allowed us to utilize immunohistochemistry (IHC), a universally available tool in diagnostic laboratories, on tissue microarrays of thousands of clinically well-annotated samples to validate ZIC2 as a prognostic protein biomarker independent of established clinicopathological variables.

\section{Results}

\section{Differential methylation analysis}

To identify methylation alterations associated with $\mathrm{PCa}$ aggressiveness, we used a discovery cohort of $70 \mathrm{PCa}$ cases (Table 1) with good (organ-confined disease and lack of recurrence for at least 5 years) or poor prognosis (systemic presence of metastatic disease, indicated by biochemical PSA-based recurrence within 3 years and no response to local radiation therapy) for genome-wide methylation analyses using Illumina $450 \mathrm{k}$ arrays. The two groups showed differences in preoperative PSA levels $\left(p=1.2 \times 10^{-6}\right)$ and survival rates. Patients in the

Table 1 Clinical characteristics of the discovery cohort

\begin{tabular}{lll}
\hline & Good prognosis & Poor prognosis \\
\hline$n$ & 35 & 35 \\
Age (mean \pm sd) & $62.7 \pm 5.6$ & $65 \pm 6.6$ \\
Pretreatment PSA (ng/ml) & $6.86 \pm 3.4$ & $28.3 \pm 22.3$ \\
Stage (path. T) & & \\
pT2 & 35 & 0 \\
pT3a & 0 & 3 \\
pT3b & 0 & 31 \\
pT4 & 0 & 1 \\
Gleason score & & \\
$3+3$ & 15 & 0 \\
$3+4$ & 17 & 6 \\
$4+3$ & 3 & 15 \\
$4+4$ & 0 & 4 \\
$4+5$ & 0 & 3 \\
$5+4$ & 0 & 7
\end{tabular}

${ }^{\mathrm{a} G o o d}$ prognosis defined as an organ-confined disease (pT2) and lack of biochemical (PSA-based) recurrence (BCR) for at least 5 years. Poor prognosis defined as systemic presence of metastatic disease, indicated by recurrence within 3 years and no response to local radiation therapy 
poor prognosis group suffered from rapid BCR, with a median disease-free survival of 3.8 months.

After adjusting for age at diagnosis and tumour purity (based on the samples' basal, stromal and immune cell contents computed from DNA methylation data using the PEPCI R-package [9] (Additional file 1: Table S1)), we selected 402 differentially methylated CpG sites (DMS, with minimum $10 \%$ absolute methylation difference, FDR-adjusted $p$ value $<0.2$ ) (Fig. 1a). Of these, 302 DMS lost methylation in the poor prognosis group compared to the good prognosis group, and 100 DMS gained methylation (Fig. 2). Hypermethylated DMS were mainly localized in CpG islands, shores and shelves, while DMS with loss in methylation were mostly located in intergenic (open sea) regions (Fig. 1b). To characterize the enrichment of DMS in specific genomic regions, we used the EpiAnnotator tool and chromatin state information (ChromHMM data) for normal prostate (PrEC) and prostate cancer (PC3, LnCAP) cell lines [9]. DMS with hypermethylation in aggressive $\mathrm{PCa}$ were enriched in poised promoters and repressed regions in normal $\mathrm{PrCE}$ cells. In both prostate cancer cell lines, these regions were marked as heterochromatin, indicating remodelling of the 3D chromatin structure during carcinogenesis. DMS that lost methylation in aggressive tumours showed enrichment for heterochromatic, often genepoor regions in normal prostate as well as in prostate cancer cell lines (Fig. 1c).

We observed only minor differences in enrichment between the androgen-responsive cell line LNCaP and ARindependent PC3 cells. Still, we explored the proximity of the genes associated with DMS to androgen receptor binding sites (ARBS), using a list of consensus ARBS $(n=8162)$ derived from Stelloo et al. [17]. For $>90 \%$ of the genes, the most proximal ARBS was located $>10 \mathrm{~kb}$ away from the transcription start sites (TSSs) (Additional file 2: Table S2), and none of the DMS directly overlapped with an ARBS. These findings indicated that androgen signalling was not the major driver underlying differential methylation between the two prognosis groups.

Poised promoters that significantly overlapped with hypermethylated DMS in PrEC synchronously bear activating and repressive histone marks at the transcription start site and are often associated with cell fate determination and differentiation [18]. In line with these observations, a GREAT-based pathway analysis [19] of genes associated with hypermethylated DMS showed enrichment of developmental processes (Fig. 1d).

\section{Random forest model}

We applied random forest-based modelling to rank the selected DMS according to their discriminative power (for details, see description in the "Methods" section). In addition to the DMS, our recently developed PurityAdjusted Epigenetic Prostate Cancer Index (PEPCI) of tumour aggressiveness [9] was included in the model. Mean PEPCI was significantly different between the two prognosis groups of the discovery cohort $(t$ test $p$ value $=$ 0.03). Using a cut-off of 69.1 to define PEPCI-low and PEPCI-high tumours (as described in [9]), the aggressivity score stratified the discovery cohort according to PSA recurrence-free survival $(\log$-rank $p$ value $=0.045)$ (Additional file 3: Figure S1).

For the random forest-based modelling, the discovery cohort was randomly split into a training ( $80 \%$ randomly selected samples) and a test set (20\% randomly selected samples). The model was trained on the training set, with 10,000 trees. Prediction accuracy was then measured on the test set. For variable selection, DMS were ranked based on mean decrease in accuracy and Gini scores [20] (complete list of CpG sites in the model, as well as importance scores in Additional file 2: Table S2). The Gini score indicates how often a random sample from the test set would be incorrectly categorized as having good or poor prognosis if the samples were randomly distributed [20].

The random forest model showed an error of $14.81 \%$ on the training set $(n=56)$, with better prediction for the poor prognosis subgroup (Additional file 4: Figure S2). On the test set $(n=14)$, the model showed an error rate of $18.8 \%$, with an area under the receiver operating characteristic (ROC) curve (AUC) of 95\% (Fig. 3a). A Kaplan-Meier plot indicated excellent stratification of the subgroups of the test set predicted to have good or poor prognosis (log-rank $p$ value $<0.0001$, Fig. $3 b)$. We applied our model to two independent PCa cohorts for validation of the good prediction rate. We were able to validate our results using the ICGC PCa cohort of earlyand late-onset prostate cancer $(n=222)$ [9]. The AUC for the sensitivity analysis was $77.1 \%$ (Fig. 3c). With an AUC of 99.7\%, the model demonstrated excellent performance when we only used a subset of the cohort based on the same selection criteria as in our discovery cohort $(n=63)$. With the TCGA PRAD cohort $(n=477$, Table 2), we observed an AUC of $68.7 \%$ (Fig. 3e), while the AUC was $77.5 \%$ with a preselected subset $(n=84$, Table 2). Our classifier efficiently stratified both validation cohorts according to PSA recurrence-free survival (log-rank $p$ value $<0.0001$ for both cohorts) (Fig. 3d, f and Additional file 5: Figure S3). In the ICGC dataset, our model proved to be an independent predictor of recurrence-free survival, when the Gleason score was included in the model (Cox regression $p=0.011$ ).

\section{Candidate selection}

Based on their localization in regulatory regions and distance from TSS, DMS were associated with genes [19]. 

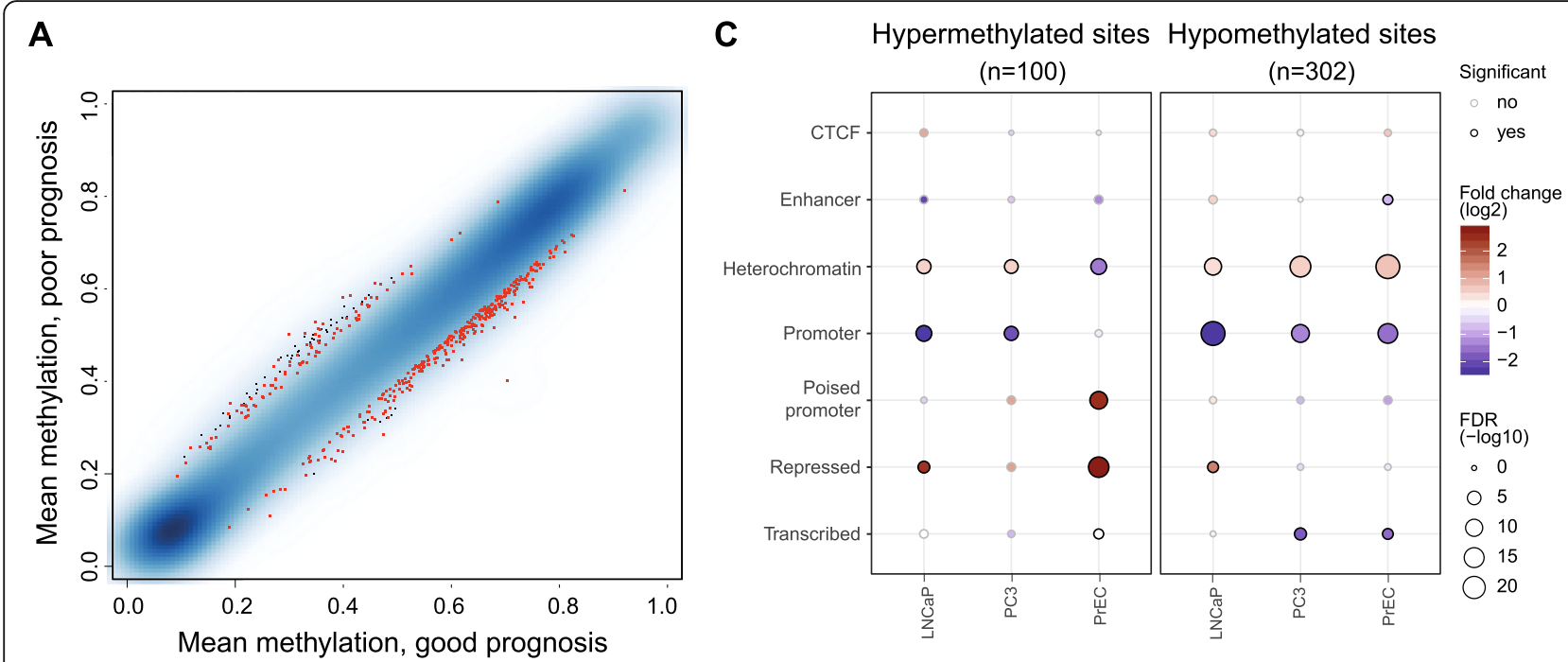

B

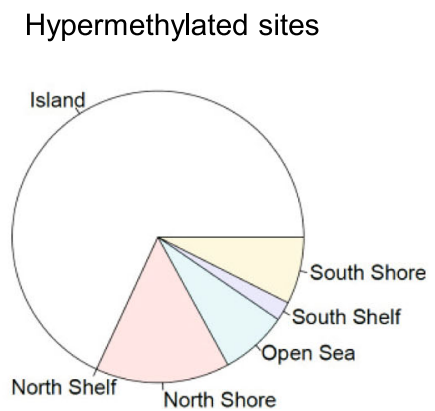

Hypomethylated sites

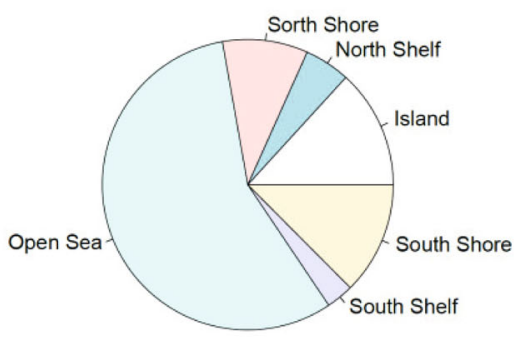

D

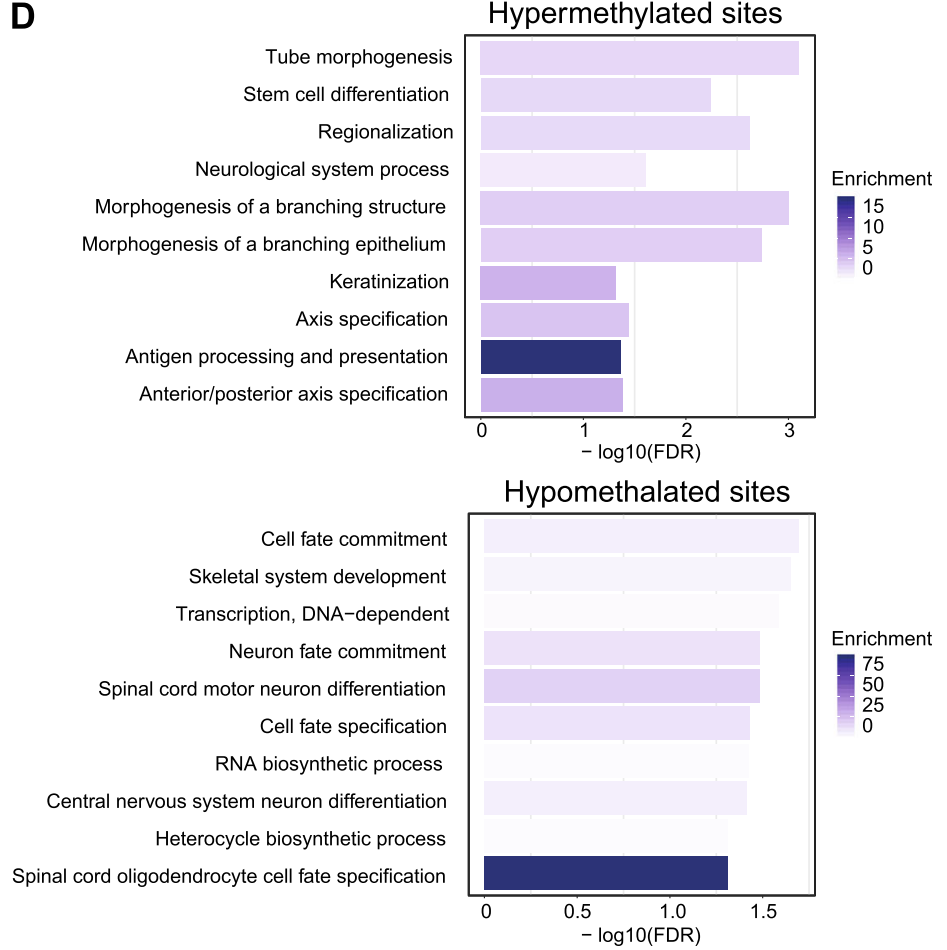

Fig. 1 Differential methylation analysis. a Mean methylation values of the good and poor prognosis groups in a smoothed colour density representation plot. Sites with FDR-corrected $p$ values $<0.2$ and absolute beta value difference $>0.1$ are marked in red. b Distribution of the localization of differentially methylated CpG sites (DMS) hypermethylated ( $n=100$, top) or hypomethylated ( $n=302$, bottom) in the poor prognosis group relative to the good prognosis group, in relation to CpG islands. $\mathbf{c}$ Enrichment analysis of the hypermethylated (left) and hypomethylated (right) DMS using 7-state ChromHMM data for PC3 and LnCaP tumour cell lines and prostate epithelial cells (PrEC) [9]. The size of the circles demonstrates the significance of enrichment, while their colour represents the strength and the direction of the enrichment (red: enriched, blue: depleted). The black circle outline indicates significant results. $\mathbf{d}$ Pathway analysis of genes associated with the hypermethylated (top) and hypomethylated (bottom) DMS. GREAT tool was used to assign genes to CpG sites. The shade of blue shows the significance of the enrichment, while the bars represent the strength

The genes were ranked to select the top 20 candidates for confirmatory analyses based on immunohistochemistry (IHC), as further described below (Table 3, with individual Kaplan-Meier curves for the candidate gene- related CpG sites and the full model in Additional file 3: Figure S1).

Comparison with recently published whole genome bisulfite sequencing data (WGBS) for prostate cancer [21] 


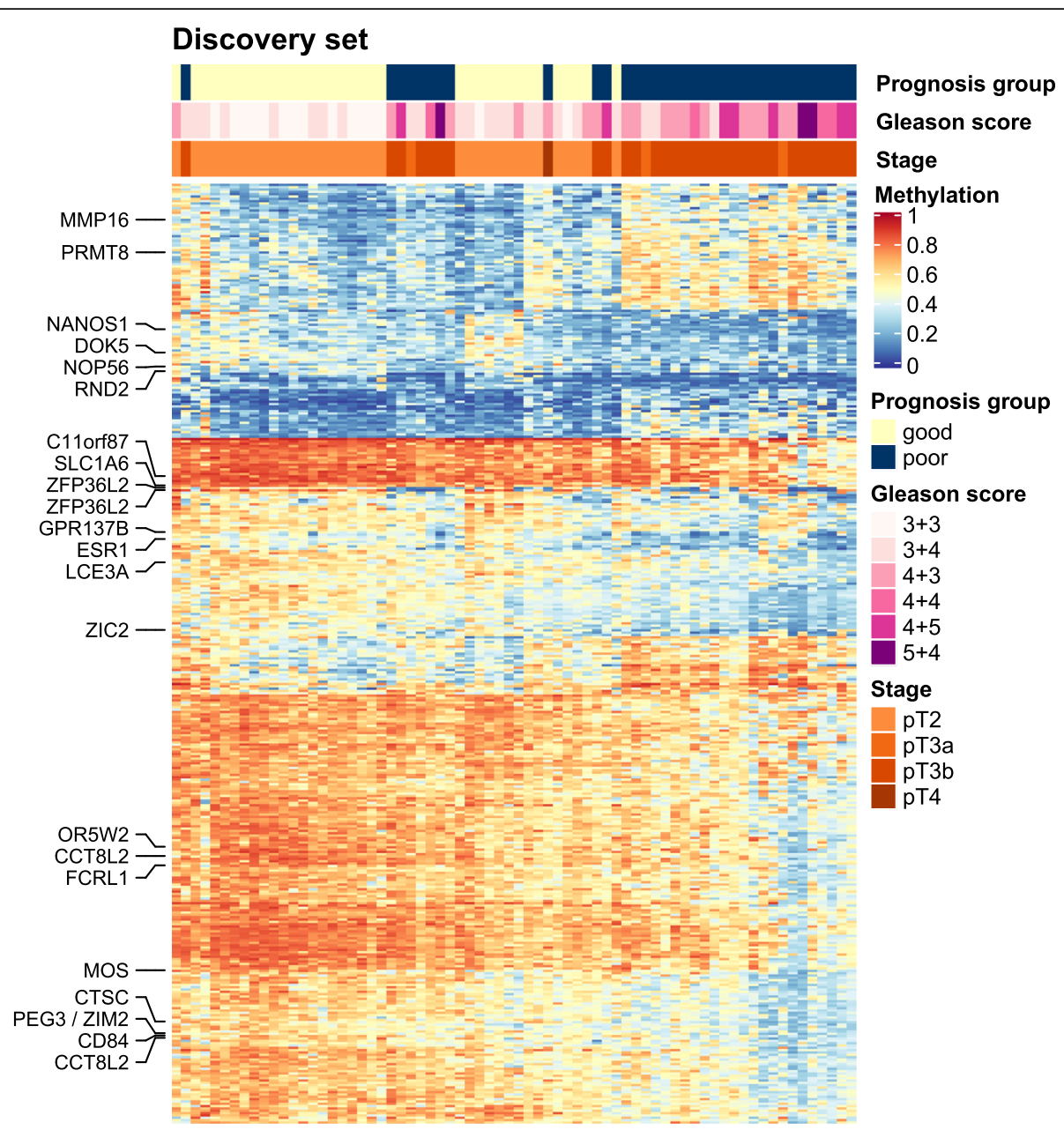

Fig. 2 Methylation heatmap of the selected CpG sites in the discovery cohort. Each column represents a sample with predicted good or poor prognosis, while rows represent selected differentially methylated CpG sites. Annotations on the left side indicate top-ranked candidate genes associated with most informative $\mathrm{CpG}$ sites. Low and high methylation beta values in a range from 0 to 1 are shown in a blue to red colour scale. Hierarchical clustering was done on Euclidian distance between samples and sites. BCR: PSA-based biochemical recurrence

revealed that about $60 \%$ of the top DMS (associated with C11orf87, CCT8L2, CD84, CTSC, DOK5, FCRL1, LCE3A, MMP16, MOS, OR5W2, PEG3/ZIM2 and SLC1A6) were located in so-called partially methylated domains (PMDs) (Additional file 6: Figure S4). PMDs are genomic regions of several hundred kilobases to few megabases in length that are associated with heterochromatic areas in the nuclear periphery, replicated late during cell cycle progression and progressively losing methylation. In tumours, stronger hypomethylation in PMDs was significantly associated with higher genome-wide somatic mutation densities [22], supporting our findings of commonly more loss of methylation in more aggressive $\mathrm{PCa}$ compared to the good prognosis group.

Beside the PMD-associated DMS, we also identified DMS with focal changes in methylation in gene promoter regions. The gene of matrix metalloproteinase 16 (MMP16), a proteolytic enzyme involved in the development of PCa progression and metastases [23], is located in a frequent PMD. However, we identified cg12818557 located in the promoter region of MMP16 as a strong predictor, with almost $20 \%$ methylation gain in the poor compared to the good prognosis group.

Gain of methylation at cg00874055 in the promoter region of GPR137B (G protein-coupled receptor 137B) was inversely correlated (rho $=-0.497, p=1.85 \times 10^{-6}$ ) with mRNA expression (data from ICGC EOPC cohort). GPR137B upregulation is linked to aggressive forms of pancreatic cancer [24] and associated with increased proliferation in various cancer types but has not been identified as a PCa biomarker yet.

Similarly, nucleolar protein NOP56 (cg18146506) and protein arginine methyltransferase PRMT8 (cg24100636) with hypomethylated promoter DMS were were identified as biomarkers for multiple cancer types [25, 26]. Arginine methylation is relevant for various cellular 


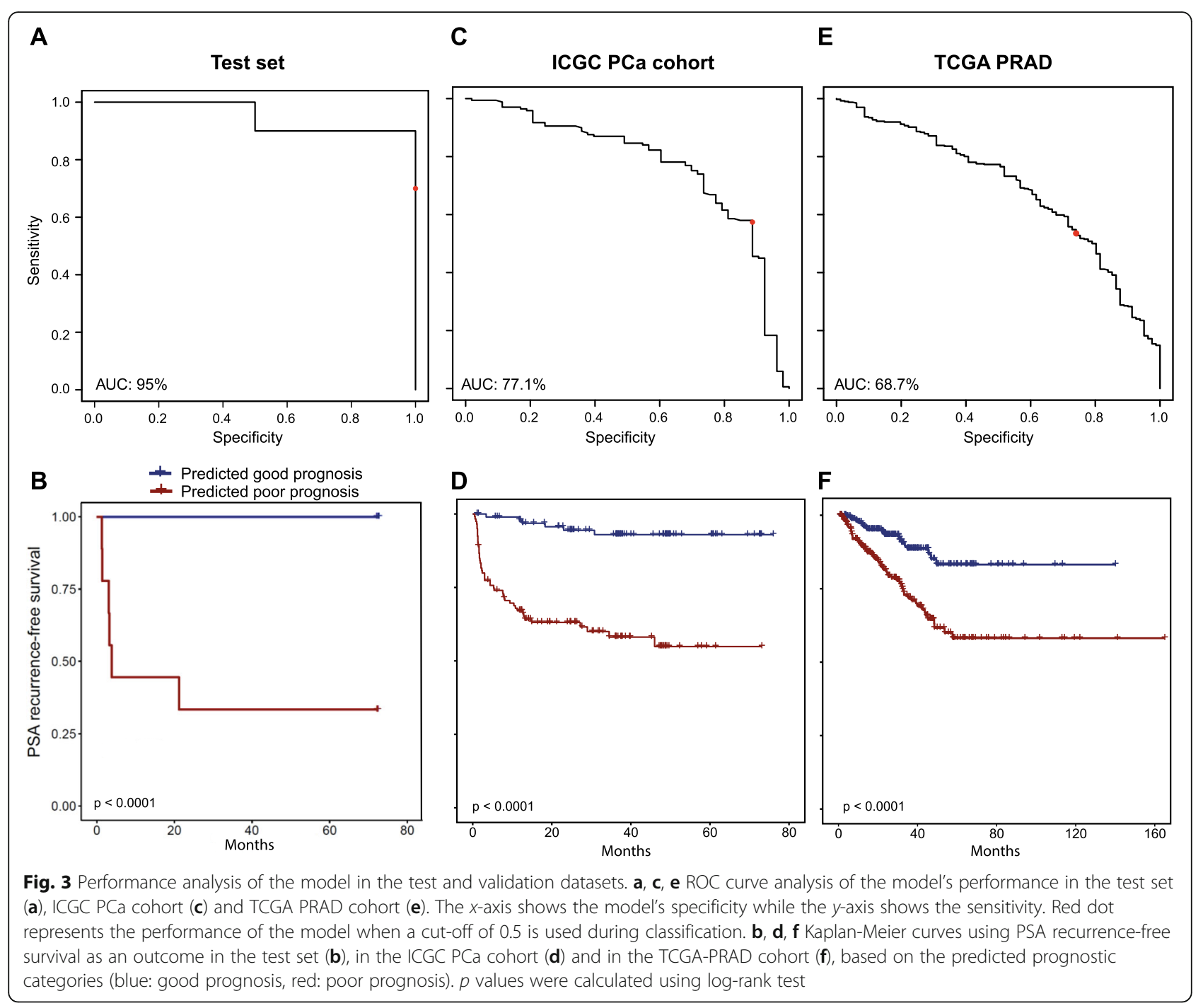

processes, including DNA repair, RNA transcription, signal transduction, protein compartmentalization, and possibly protein translation [27]. We also identified a DMS (cg17225407) in the promoter of $R N D 2$, a relatively unexplored member of the Rho GTPase family [28], with loss in methylation in the poor prognosis group compared to the good prognosis group. Cg04211581 is located only $26 \mathrm{bps}$ from the TSS of ESR1. ESR1 encodes oestrogen receptor alpha $(E R \alpha)$, the role of which has been proposed in $\mathrm{PCa}$; however, it is still controversial [29]. Interestingly, we identified an ARBS in close vicinity (distance $<1 \mathrm{kbp}$ ) of the DMS.

Three DMS affected the promoter region of zinc finger proteins. Two sites were located in the promoter of ZFP36L2 (cg16876647, cg12092201), while one CpG site (cg24690071) was located in a poised promoter of ZIC2. ZFP36L2 encodes a CCH-type zinc finger protein, which is regulated by the cell-cycle, might play a role in DNA damage response [30] and inhibit cell proliferation [31].
In PCa, ZFP36L2 upregulation was associated with the transcription factor Runx2 and poor prognosis [32]. ZIC2 belongs to a family of transcription factors involved in neuroectodermal development. Elevated ZIC2 mRNA expression was described in high Gleason prostate cancer [33].

Our results highlight the prognostic relevance of methylation loss in PMD regions, as well as of several candidate genes not previously associated with PCa. The influence of the methylation changes of these candidates DMS on gene or protein expression and the impact on prostate carcinogenesis needs to be experimentally confirmed in mechanistic chromatin conformation and gainand loss-of-function studies.

\section{Candidate validation}

ZIC2 was one of the candidate genes for which a suitable antibody for IHC was available. ZIC2 expression was analysed by immunohistochemistry on a tissue 
Table 2 Clinical characteristics of the TCGA PRAD cohort and the preselected subcohort

\begin{tabular}{|c|c|c|c|}
\hline & \multirow{2}{*}{$\begin{array}{l}\text { TCGA PRAD } \\
\text { Full cohort }\end{array}$} & \multicolumn{2}{|c|}{ TCGA PRAD subcohort } \\
\hline & & Good prognosis $^{a}$ & Poor prognosis ${ }^{a}$ \\
\hline$n$ & 477 & 27 & 57 \\
\hline Age (mean \pm sd) & $61.06 \pm 6.9$ & $57.23 \pm 6.7$ & $62.46 \pm 6$ \\
\hline \multicolumn{4}{|l|}{ Stage (path. T) } \\
\hline n.a. & 6 & 0 & 0 \\
\hline pT2a & 13 & 1 & 0 \\
\hline pT2b & 6 & 3 & 0 \\
\hline pT2c & 161 & 23 & 0 \\
\hline рT3a & 153 & 0 & 24 \\
\hline pT3b & 129 & 0 & 31 \\
\hline pT4 & 9 & 0 & 2 \\
\hline \multicolumn{4}{|l|}{ Gleason score } \\
\hline 6 & 43 & 6 & 0 \\
\hline 7 & 243 & 17 & 12 \\
\hline 8 & 61 & 2 & 8 \\
\hline 9 & 127 & 2 & 36 \\
\hline 10 & 3 & 0 & 1 \\
\hline
\end{tabular}

n.a. not available

${ }^{\mathrm{a}}$ Good prognosis defined as lack of BCR for at least 5 years and stage $<$ pT3. Poor prognosis defined as BCR within 3 years and stage $\geq \mathrm{pT} 3$

microarray (TMA) containing more than 12,000 prostate cancer specimens (Table 4). Results were compared with tumour phenotype, BCR, ETS-related gene (ERG) status and other recurrent genomic alterations. ZIC2 expression was detectable and considered to be strong in $23.3 \%$ of cases and was absent in the majority of the tumours (76.7\%) (Fig. 4a, Table 4). Loss of ZIC2 protein expression was associated with ERG-fusion positivity $(p<0.0001)$ (Fig. 4b). Loss of ZIC2 expression was also linked to Gleason grade, advanced pathological tumour (pT) stage, lymph node metastasis and higher preoperative PSA levels in all cancers $(p<0.0001$, each) and in the subset of ERG-fusion negative tumours (Table 4, data not shown). These associations were either weaker or absent in ERG-fusion positive cancers (data not shown). Within ERG fusion-negative cancers, ZIC2 expression was also strongly associated with $6 \mathrm{q} 15$ and 5 q21 deletions $(p<0.001)$ (Fig. 4c). Loss of ZIC2 expression was associated with adverse outcome and correlated with significantly shorter time to biochemical recurrence in all cancers, independent of ERG and PTEN (Fig. 4d). The prognostic value of ZIC2 proved to be independent from established clinicopathological variables including Gleason, stage, nodal stage and PSA. Overall, ZIC2 was identified as an excellent marker and might provide clinically useful predictive information by identification of aggressive prostate cancer subsets.

\section{Discussion}

In the present study, we have identified methylation differences related to $\mathrm{PCa}$ prognosis and subsequently showed that methylation-based prediction of $\mathrm{PCa}$ prognosis using random forest-based modelling is feasible with high accuracy.

$\mathrm{PCa}$ is the most prevalent cancer among men in Germany. With a 5 -year survival rate of $91 \%, \mathrm{PCa}$ is a cancer type with comparably good prognosis (German Cancer Registry). Nevertheless, biomarkers predicting the prognosis of $\mathrm{PCa}$ are needed for an efficient clinical management, to avoid overtreatment of cases with indolent disease and to identify patients who develop aggressive forms and require chemotherapy [34].

DNA methylation is an excellent source for biomarker development, since it is a stable modification and can be quantitatively determined in clinical samples with high throughput and precision and relatively low cost [35]. Previous studies trying to establish a methylation-based classifier for prostate cancer mostly used a preselected set of genes [36, 37] or used high Gleason score as an outcome [38, 39]. Here, we are presenting a genomewide approach, with PSA recurrence-free survival as an endpoint. One limitation of our study is the use of the Illumina 450k platform for biomarker selection, which limits methylation analyses to preselected $\mathrm{CpG}$ sites on the $450 \mathrm{k}$ array (enriched for CpG islands and flanking regions, bioinformatically predicted enhancers, DNase I hypersensitive sites, and validated differentially methylated regions [40]). Future studies using whole genome bisulfite sequencing (WGBS) of all > 29 million CpG sites in the human genome will allow identification of additional biomarkers.

Our discovery cohort consisted of 70 patients, 35 with good and 35 with poor prognosis. After cell type adjustments, our cut-off criteria for selection of differentially methylated CpG sites were absolute methylation differences $>10 \%$ and an FDR-adjusted $p$ value < 0.2. Altogether, 402 DMS and the PEPCI score for tumour aggressiveness [9] were included in the prediction model. Our random forest-based model demonstrated excellent performance with the discovery cohort (AUC 95\%). We were able to validate our results using the ICGC PCa cohort of early and late prostate cancer (AUC 77.1\%), with slightly worse performance using the TCGA PRAD dataset (AUC $68.7 \%$ ). Different reasons might contribute to the lower performance with the TCGA PRAD cohort, such as possibly different definitions of PSA recurrence-free survival and the generally high Gleason score and high tumour stage of the TCGA patients. Other genomewide studies have faced similar problems using TCGA as a validation set $[38,41]$. Nevertheless, for both ICGC and TCGA validation cohorts, the resulting 


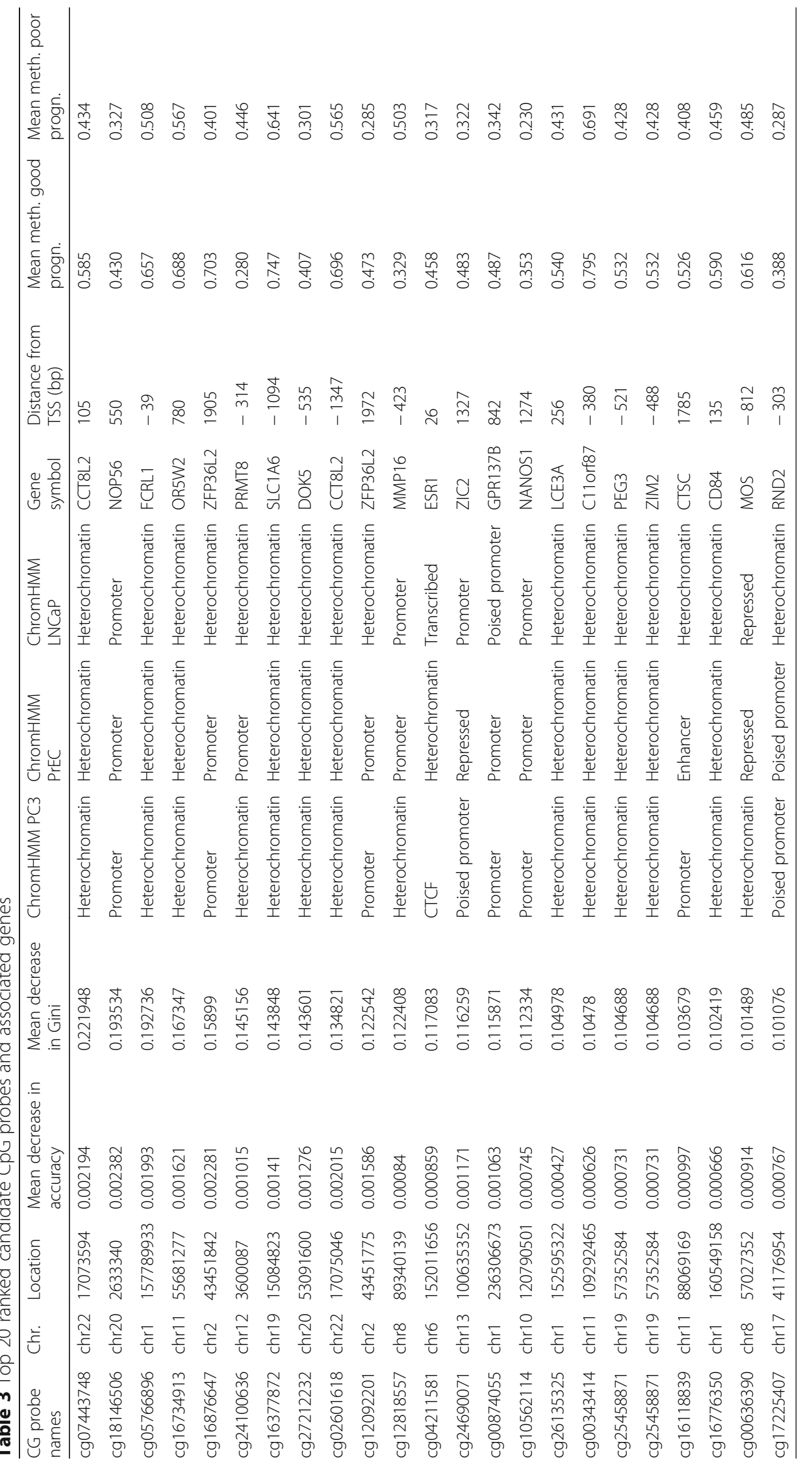


Table 4 Association between ZIC2 immunostaining results and prostate cancer phenotype in tissue micro-arrayed cancers

\begin{tabular}{|c|c|c|c|c|c|}
\hline Parameter & $n$ evaluable & Negative (\%) & Positive (\%) & $p$ value & Bonferroni correction \\
\hline All cancers & 12,581 & 76.7 & 23.3 & & \\
\hline \multicolumn{6}{|l|}{ Tumour stage } \\
\hline pT2 & 7994 & 74.6 & 25.4 & \multirow[t]{3}{*}{$<0.0001$} & 0.000006 \\
\hline рТ3а & 2837 & 78.7 & 21.3 & & 0.000018 \\
\hline pT3b-pT4 & 1700 & 83.1 & 16.9 & & 0.000029 \\
\hline \multicolumn{6}{|l|}{ Gleason grade } \\
\hline$\leq 3+3$ & 2303 & 71.5 & 28.5 & \multirow[t]{6}{*}{$<0.0001$} & 0.000022 \\
\hline $3+4$ & 6700 & 75.1 & 24.9 & & 0.000007 \\
\hline $3+4$ Tert. 5 & 606 & 77.7 & 22.3 & & 0.000083 \\
\hline $4+3$ & 1238 & 81.4 & 18.6 & & 0.000040 \\
\hline $4+3$ Tert. 5 & 907 & 85.6 & 14.4 & & 0.000055 \\
\hline$\geq 4+4$ & 731 & 87.1 & 12.9 & & 0.000068 \\
\hline \multicolumn{6}{|c|}{ Lymph node metastasis } \\
\hline NO & 7459 & 77.6 & 22.4 & \multirow[t]{2}{*}{$<0.0001$} & 0.000007 \\
\hline $\mathrm{N}+$ & 932 & 86.3 & 13.7 & & 0.000054 \\
\hline \multicolumn{6}{|c|}{ Preop. PSA level (ng/ml) } \\
\hline$<4$ & 1479 & 75.8 & 24.2 & \multirow[t]{4}{*}{$<0.0001$} & 0.000034 \\
\hline $4-10$ & 7470 & 75.4 & 24.6 & & 0.000007 \\
\hline $10-20$ & 2642 & 79.2 & 20.8 & & 0.000019 \\
\hline$>20$ & 916 & 81.3 & 18.7 & & 0.000055 \\
\hline \multicolumn{6}{|c|}{ Surgical margin } \\
\hline Negative & 10,018 & 75.8 & 24.2 & \multirow[t]{2}{*}{$<0.0001$} & 0.000005 \\
\hline Positive & 2519 & 80.3 & 19.7 & & 0.000020 \\
\hline
\end{tabular}

prognostic subgroups had highly significantly different survival rates.

We compared the performance of our model with commercially available, RNA expression-based genomic tests. Using RNA-seq data available for the TCGA PRAD cohort, we generated sums of $Z$-scores for the gene lists included in the Decipher, OncotypeDX and Prolaris tests, as described by Wei et al. [42]. Prolaris outperformed Decipher and OncotpyeDX with an AUC of $64.6 \%$ versus $51.5 \%$ and $50.8 \%$ for Decipher and OncotypeDX (Additional file 7: Figure S5). Our methylation-based classifier showed a higher AUC (68.7\%) with the TCGA PRAD cohort. The low performance of Decipher and OncotypeDX might be due to the fact that the commercially available tests were not designed for RNA-seq data.

We identified significantly more loss than gain in methylation associated with $\mathrm{PCa}$ progression and could map the majority of our top selected candidate biomarker to PMDs. A recent small breast cancer study concluded that loss of methylation in PMDs might be more valuable as diagnostic than prognostic biomarker [43]. Generally, loss of methylation of candidate DMS located in PMDs might be more informative on larger- scale methylation changes in these late-replicating heterochromatic regions than have functional relevance on the expression of the associated genes. Accordingly, Brinkman et al. concluded that PMDs commonly did not overlap with tumour suppressor genes in breast cancer [43]. Our findings, in conjunction with WGBS data on PCa [21], support a more intensive analysis of the prognostic relevance of PMD methylation in PCa.

A recent proteomics-based biomarker study of curable prostate cancer reported a stronger link of DNA methylation status to protein than mRNA abundance [44]. In line with these findings, we performed a validation of the clinical impact of ZIC2 as one of the candidate genes on more than 12,000 micro-arrayed PCa cases. The zinc finger of the cerebellum (ZIC) family of genes consists of five human homologues ZIC1-5 [45]. ZIC family members inhibit TCF4/ $\beta$-catenin and interact with GLI signalling [46]. ZIC2 is related to the sonic hedgehog pathway. Its oncogenic role was described in epithelial ovarian cancer [47], hepatocellular carcinoma [48] and pancreatic cancer [49]. Our IHC validation indicated a particularly strong adverse prognostic value of ZIC2 expression loss, including early biochemical recurrence and high Gleason grade. Of note, an eminent weakness 


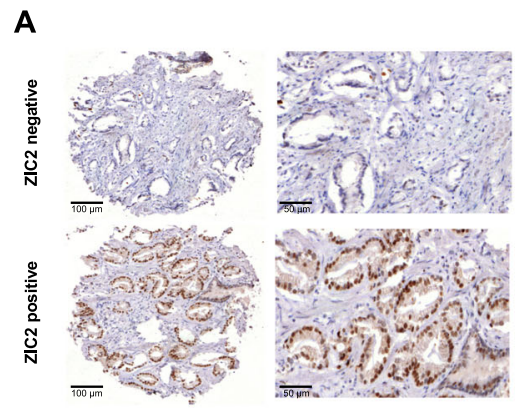

B

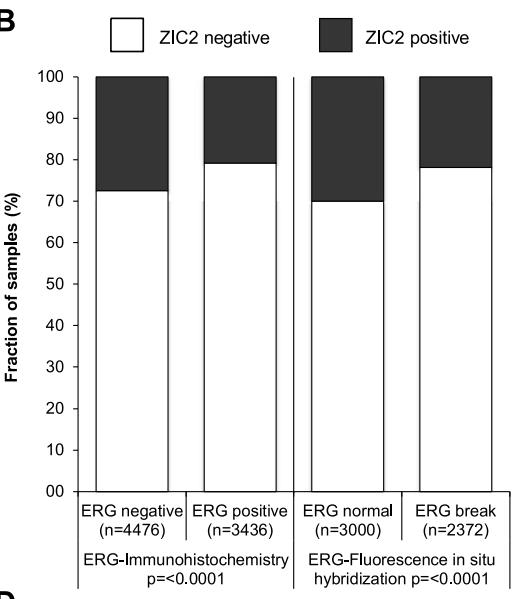

C a) all tumors

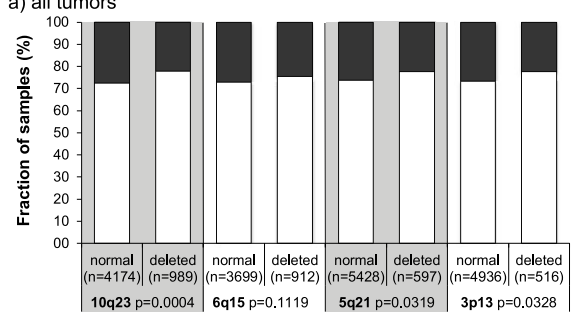

b) ERG fusion negative

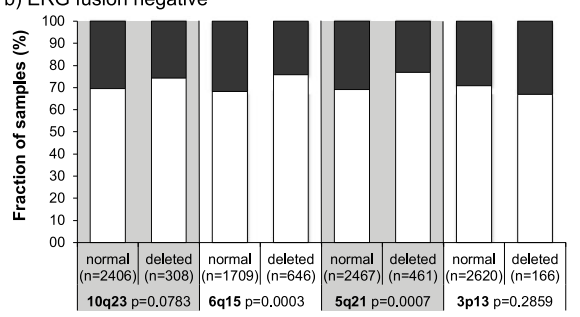

c) ERG fusion positive

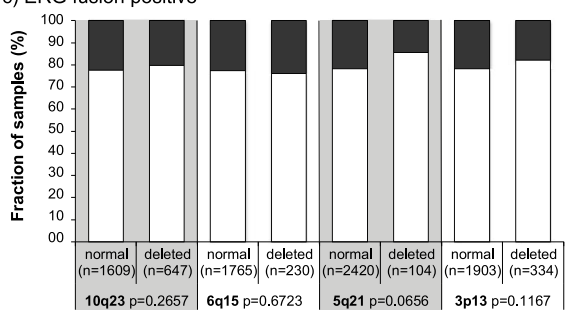

a) all tumors

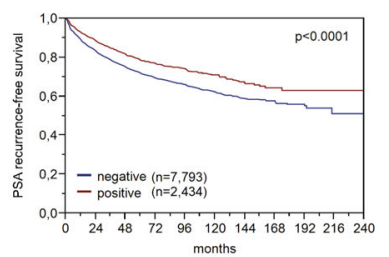

months

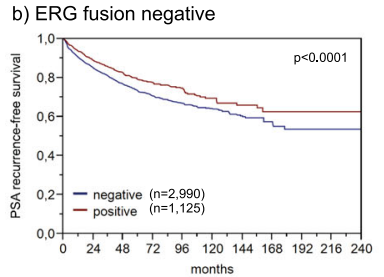

c) ERG fusion positive

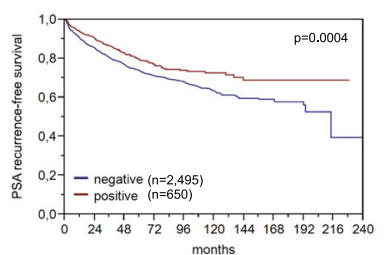

months

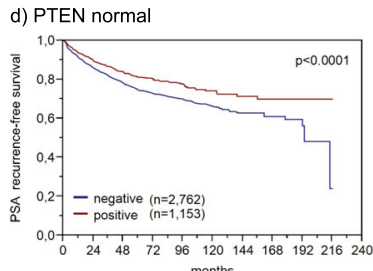

e) PTEN deleted

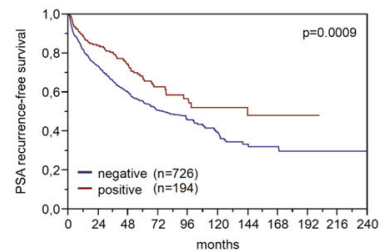

months

Fig. 4 ZIC2 immunostaining in > 12,000 micro-arrayed PCa cases. a Examples of negative (no nuclear staining, upper panels) and strong staining (lower panels). b Association between ZIC2 immunostaining results and the ERG-status determined by IHC and FISH analysis. c Association between ZIC2 immunostaining and deletions of 10q23 (PTEN), 6 q15 (MAP 3 K7), 5 q21 (CHD1) and 3p13 (FOXP1) for all cancers (a), ERG fusionnegative (b) and ERG fusion-positive subset (c) according to ERG-IHC analysis. d Kaplan-Meier curves for the relationship of ZIC2 immunostaining with PSA recurrence-free survival in all cancers (a), in ERG fusion-negative cancers (b), in ERG fusion-positive cancers (c), in PTEN normal cancers (d) and in PTEN deleted cancers (e). Log-rank $p$ values

of Gleason grading is the high inter-observer variability between pathologists that generally exceeds 30\% [50]. In this study, the original Gleason grade from the patient's files was used for statistical analyses. From 2005 on, in our department, Gleason grading was performed almost exactly as recommended by the WHO 2016 classification [51]. ZIC2 analysis, thus, appears to be of high value for distinguishing between patients with more or less aggressive forms of the disease and may be useful to select patients for active surveillance.

There are some limitations connected to our study. The patient cohort used for candidate gene identification was selected from patients subjected to curative radical prostatectomy and did not include individuals with 
advanced castration-resistant cancers who have the worst prognosis. Also, a 5-year recurrence-free interval as defined for our good prognosis group might be too short to select only patients with the best possible prognosis. Thus, it cannot be excluded that some relevant candidate genes may have been missed by our approach. The same might also apply to the 17,000 cancer validation set, which is also limited to prostatectomy specimens. An optimal validation set would have been made up from biopsy specimens which precisely represent the kind of samples that are available for molecular analysis when a therapy decision has to be taken. However, needle biopsies are precious material that is exhausted after only a few analyses. The 0.6-mm TMA punches used in our study very much resemble the size of needle biopsies. This makes them probably well-suited to reflect the diagnostic problems connected to needle biopsy analysis including a possible selection bias, heterogeneity issues and the limited amount of cancer cells available for analysis.

\section{Conclusions}

We present a candidate selection of cancer progressionrelated CpG methylation changes, as well as a classification model to predict aggressive behaviour of PCa. This model, with further tuning, might help in decision making related to the treatment of prostate cancer patients. The effect of candidate CpG site methylation on gene expression helps to pinpoint further genes, which play an important role in prostate cancer development. Ranking of the selected $\mathrm{CpG}$ sites and associated genes allowed selection of candidate biomarkers for validation by IHC. We identified loss of ZIC2 expression as a promising prognostic biomarker for $\mathrm{PCa}$.

\section{Methods}

\section{Study population}

In order to build a classifier that predicts the patients' outcome the best, a highly selected group of patients was included in the study. Sample selection was based on the following criteria: good prognosis indicated by the presence of organ-confined disease (pT2) and lack of biochemical prostate-specific antigen (PSA)-based recurrence (BCR) for at least 5 years. In contrast, poor prognosis is defined as systemic presence of metastatic disease, indicated by BCR within 3 years and no response to local radiation therapy. Initially, 84 patients were selected.

A pathologist selected FFPE tissue blocks containing tumour-rich areas ( $\geq 70 \%$ tumour cells) for analysis. Three tissue punches $(0.6 \mathrm{~mm} \times 3 \mathrm{~mm})$ were taken of each tissue block, and genomic DNA was isolated using the AllPrep ${ }^{\circ}$ DNA/RNA FFPE kit (Qiagen). DNA was submitted to the DKFZ Genome and Proteome core facility for Illumina 450k Methylation analyses. After removing samples and DNA methylation profiles with low quality, the study included 35 patients with good and 35 patients with bad prognosis (Additional file 1: Table S1).

\section{Validation datasets}

The ICGC PCa cohort has been described earlier [9]. Clinical information for the TCGA PRAD cohort was downloaded from cBioPortal in June 2018 (Table 2). For the subcohorts, patients were selected as good prognosis patients by lack of BCR within 5 years and a disease stage pT2 and as poor prognosis patients when suffering from BCR within 3 years and having a stage pT3 or pT4. Consensus androgen receptor (AR) binding sites $(n=$ 8162) were defined by Stelloo et al. [17] based on AR ChIP-seq data for 100 prostate carcinomas. Genomic distances of DMS-associated gene TSSs were calculated using the middle point of the nearest region. Prostate cancer WGBS data was accessed at GEO accession number GSE104789 [21]. Information on common PMDs was derived from [22].

\section{DNA methylation processing}

DNA methylation was assessed using the Illumina HumanMethylation450 Array. The methylation data was processed using the RnBeads $\mathrm{R}$ package [52]. Probes with SNPs (dbSNP 144) overlapping with the C nucleotide of the CG site and having MAF>0.01 (28,722 probes) were excluded. Probes with high likelihood of false hybridization (28,736 probes, as defined in RnBeads) were also removed. Quality filtering was performed using the Greedycut algorithm, which removed 21,040 probes and 11 samples. Additional 969 non-CpG probes and 9229 probes located on the sex chromosomes were removed. No normalization or background correction was used.

During the analysis, a batch effect was observed between data from fresh frozen (TCGA PRAD and ICGC PCa cohort) and formalin-fixed tissue (discovery cohort). In order to have a generalizable model, we avoided shifting the beta values as would happen with batch correction methods. Instead, we used principal component analysis (PCA) on the top 10,000 most variable CpG sites to identify the probes affected by this effect. This was done using two independent datasets containing formalin-fixed [53] or fresh frozen tissue [8] and removed the top 5000 sites captured by PC2, the main principal component affected by the sample type. The PEPCI score and the basal, stromal, luminal, T-luminal and immune cell composition were estimated using the PEPCI R package [9]. Linear models of the limma package [54] were applied to identify differentially methylated probes after adjustments for age, basal, stromal and immune cell content. CpG sites with FDR-adjusted $p$ values 
$<0.2$ and mean methylation difference $>0.1(10 \%)$ were used to build the model. Enrichment analysis of the significantly methylated sites, promoters and genes were performed with EpiAnnotator [55]. Annotation of the most important $\mathrm{CpG}$ sites of the random forest model was done using the GREAT tool [19].

\section{Random forest classifier}

A random forest-based classifier was built using the randomForest $\mathrm{R}$ package, which is based on the algorithm of Breiman and Cutler [16]. Random forest is a learning method that constructs numerous decision trees and outputs the classes (in case of classification) of the individual trees. The predicted class of the input instance will be decided upon majority vote (schematic principle in Additional file 8: Figure S6). Each tree was built on a bootstrap training set, which represents about two thirds of the discovery cohort with replacement. Out-of-bag (OOB) error was used to measure the performance of the model on the training set. Classification of the instances left out (OOB samples) was used to estimate a generalization error (OOB error). The $\mathrm{OOB}$ error will give an unbiased estimate of the current classification error, while the bagging method will decrease the chance of overfitting.

Two variable importance scores are used in random forest. The mean decrease in accuracy reflects a variable importance measure to assess the prediction strength of each predictor variable. When a tree is grown, the OOB samples are used to calculate the error rate. Then, the values of a given predictor variable are randomly permuted and the error rate is calculated again. The decrease in accuracy caused by the permutation is averaged over all trees. The mean decrease in Gini score gives the improvement in the split-criterion at each split in each tree [20].

Twenty different models were trained as follows: data was randomly split into training $(80 \%)$ and test $(20 \%)$ set. The model was trained on the training set, with 10 , 000 trees and 19 variables to select randomly for each tree. Prediction accuracy was measured on the test set. The results were collected and the best performing model was selected. This model was then optimized for the number of variables selected for each tree. For variable selection, $\mathrm{CpG}$ sites were ranked based on mean decrease in accuracy and mean decrease in Gini scores [20].

Validation of the classifier was performed using the TCGA PRAD and the ICGC cohort of early and late prostate cancer. TCGA-PRAD DNA methylation data was downloaded from the GDC portal (https://portal. gdc.cancer.gov/) legacy archive in .idat format. The performance was evaluated with ROC curve analysis, using the ROCR $\mathrm{R}$ package [56] and Kaplan-Meier curves for the validation datasets and individual candidate CpG sites.

\section{Candidate selection}

Based on our model, the top-rated candidates underwent a further selection to identify the ones with the highest possibility to perform well as a protein expression-based biomarker. First, we used the GREAT tool and the gene annotation of the Illumina 450k methylation array to identify gene-CpG site associations, by selecting the genes closest to the sites. The CpG sites in close vicinity to transcription start sites $( \pm 2 \mathrm{~kb})$ were preferred, to enhance the potential functional relevance for correlated gene/protein expression changes. Finally, the selection was based on the mean decrease in the Gini score (cutoff $>0.1$ ).

\section{Genomic risk scores}

Risk scores for TCGA-PRAD based on the gene expression panels of Decipher, Oncotype DX and Prolaris tests were calculated as described in [42]. TCGA-PRAD RNA-Seq HTSeq counts were downloaded from GDC portal (https://portal.gdc.cancer.gov/). Gene-based Zscores were calculated for the 19, 12 and 31 genes of the respective panels. The sum of the scores was used as risk scores.

\section{Validation of candidate genes by immunohistochemistry (IHC) \\ Patients}

Radical prostatectomy specimens were available from 17, 747 patients undergoing surgery between 1992 and 2017 at the Department of Urology and the Martini Clinics at the University Medical Centre Hamburg-Eppendorf (Additional file 9: Table S3). All prostate specimens were analysed according to a standard procedure, including complete embedding of the entire prostate for histological analysis [57]. Histo-pathological data was retrieved from the patient files, including tumour stage, Gleason grade, nodal stage and resection margin status. Gleason grading was performed already from 2005 on as outlined later in the $2016 \mathrm{WHO}$ recommendations with minor modifications, i.e., we have a conservative position to define irregular glands as Gleason 4. Follow-up data were available for a total of 14,464 patients with a median follow-up of 48 months (range 1 to 241 months; Additional file 9: Table S3). PSA values were measured in regular intervals following surgery, and PSA recurrence was defined as the measurement of a postoperative PSA of $\geq 0.2 \mathrm{ng} / \mathrm{ml}$ and increasing. The TMA manufacturing process was described in detail earlier [58]. In short, one $0.6-\mathrm{mm}$ core was taken from a tumourcontaining tissue block from each patient. The molecular database attached to this TMA contained results on 
ERG expression in 10,711 [3], ERG break apart FISH analysis in 7122 (expanded from [59]), deletion status of $5 \mathrm{q} 21$ (CHD1) in 7932 (expanded from [60]), 6q15 (MAP 3 K7) in 6069 (expanded from [61]), 10q23 $(P T E N)$ in 6704 (expanded from [62]) and 3p13 (FOXP1) in 7081 (expanded from [63]) cancers.

\section{Immunohistochemistry}

Freshly cut TMA sections were immunostained on one day and in one experiment. Slides were deparaffinised and exposed to heat-induced antigen retrieval for $5 \mathrm{~min}$ in an autoclave at $121^{\circ} \mathrm{C}$ in $\mathrm{pH} 7.8$ Tris-EDTA-citrate buffer. The primary antibody specific for ZIC2 (antibodies online, ABIN2776475) was applied at $37^{\circ} \mathrm{C}$ for $60 \mathrm{~min}$. Bound antibody was then visualized using the EnVision Kit (Dako, Glostrup, Denmark) according to the manufacturer's directions. ZIC2 staining intensity was assessed as negative or positive.

\section{Statistics}

Statistical calculations were performed with JPM 12 software (SAS Institute Inc., NC, USA). Contingency tables and the $\chi^{2}$ test were performed to search for associations between molecular parameters and tumour phenotype. Survival curves were calculated according to KaplanMeier. The log-rank test was applied to detect significant survival differences between groups. Cox proportional hazards regression analysis was performed to test the statistical independence and significance between pathological, molecular and clinical variables. Separate multivariate analyses were performed using different sets of parameters available either before or after prostatectomy.

\section{Supplementary information files}

Supplementary information accompanies this paper at https://doi.org/10. 1186/s13148-019-0736-8

\section{Additional file 1: Table S1. Clinical information and cell type composition of discovery cohort samples.}

Additional file 2: Table S2. Full list of $\mathrm{CpG}$ sites contributing to the model.

Additional file 3: Figure S1. Individual Kaplan-Meier curves. Predictive power for PSA recurrence-free survival of the full classifier, PEPCI, and individual candidate $\mathrm{CpG}$ sites associated with the top20 selected genes in the discovery cohort $(n=70)$. $p$ values from log-rank test. Red: high methylation (above median), blue: low methylation (below median),

Additional file 4: Figure S2. Performance of the random forest model. The plot shows the performance of the random forest model as a function of the trees built in the model, using the generalized $\mathrm{OOB}$ (black) and classification error for the good (red) and poor (green) prognosis groups.

Additional file 5: Figure S3. Heatmap of the selected $\mathrm{CpG}$ sites in the ICGC prostate cancer (left) and TCGA PRAD (right) validation datasets. Each column represents a sample with predicted good or poor prognosis, while rows represent selected differentially methylated CpG sites. Annotations on the left side indicate top ranked candidate genes associated with most informative CpG sites. Low and high methylation beta values in a range from 0 to 1 are shown in a blue to red color scale. BCR: PSA-based biochemical recurrence.

Additional file 6: Figure S4. Localization of DMS in PMDs identified in prostate cancer by WGBS. WGBS data for three prostate cancer cases with matching benign tissue was derived from GSE104789 and uploaded to the UCSC genome browser. For comparison, common PMDs identified in eight common cancer types excluding prostate cancer [22] were displays in a color gradient from light grey to black.

Additional file 7: Figure S5. Specificity and sensitivity of gene expression-based prognostic tests to prognosticate PSA-based BCR for the TCGA PRAD cohort. Sums of Z-scores of RNA-seq-derived gene expression per patient were used for calculations of risk scores, as described in Ref. [42].

Additional file 8: Figure S6. Schematic representation of the random forest model.

Additional file 9: Table S3. Pathological and clinical data of the arrayed prostate cancers.

\section{Abbreviations}

AUC: Area under the receiver operating characteristic (ROC) curve; BCR: Biochemical recurrence; DMS: Differentially methylated $\mathrm{CpG}$ site; FFPE: Formalin-fixed paraffin-embedded; IHC: Immunohistochemistry; OOB: Out-of-bag; PCa: Prostate cancer; PMDs: Partially methylated domain; PSA: Prostate-specific antigen; ROC: Receiver operating characteristic; TMA: Tissue micro-array; TSS: Transcription start site; ZIC2: Zic family member 2

\section{Acknowledgements}

We thank the patients and families who contributed to this study. The authors acknowledge the DKFZ Genomics and Proteomics Core Facilities for excellent service and Karin Klimo and Marion Bähr (DKFZ, Division Cancer Epigenomics) for excellent technical assistance.

\section{Author contributions}

$C G, C P, R S$ and $G S$ conceived the project. Supervision was given by $Y A, C P$, CG, SM and RS. Samples were provided by TS and JM. Experiments were performed by HS, CHM, FB, DH, SW, PL and CF. RS and RT performed data analysis, RT and RS visualized the results. RT, RS and CG wrote the manuscript. All authors read and approved the final manuscript.

\section{Funding}

The study was funded by a grant from the Sander Stiftung (no. 2015.010.1) awarded to CP and GS.

\section{Availability of data and materials}

Methylation data for the discovery cohort has been uploaded to GEO under accession No. GSE127985.

\section{Ethics approval and consent to participate}

All PCa samples had been collected between 1992 and 2012 from routine diagnosis leftover tissues, the usage of which for research purposes is legally covered by $\S 12$ of the Hamburgisches Krankenhausgesetz. The local ethics committee approved the usage of these tissues for TMA manufacturing (WF049/09, Ethik-Kommision der Ärztekammer Hamburg).

\section{Consent for publication}

Not applicable.

\section{Competing interests}

The authors declare that they have no competing interests.

\section{Author details}

'Cancer Epigenomics, German Cancer Research Center (DKFZ), 69120 Heidelberg, Germany. ${ }^{2}$ Department of Pathology, University Medical Center Hamburg-Eppendorf, 20246 Hamburg, Germany. ${ }^{3}$ Department of Urology, Charité Universitätsmedizin Berlin, 10117 Berlin, Germany. ${ }^{4}$ German Cancer Consortium (DKTK), 69120 Heidelberg, Germany. ${ }^{5}$ General, Visceral and 
Thoracic Surgery Department and Clinic, University Medical Center Hamburg-Eppendorf, 20246 Hamburg, Germany.

\section{Received: 28 May 2019 Accepted: 3 September 2019 Published online: 22 October 2019}

\section{References}

1. Bray F, Ferlay J, Soerjomataram I, Siegel RL, Torre LA, Jemal A. Global cancer statistics 2018: GLOBOCAN estimates of incidence and mortality worldwide for 36 cancers in 185 countries. CA Cancer J Clin. 2018;68(6):394-424.

2. Gelmann EP. Complexities of prostate-cancer risk. N Engl J Med. 2008;358(9): 961-3.

3. Weischenfeldt J, Simon R, Feuerbach L, Schlangen K, Weichenhan D, Minner $S$, et al. Integrative genomic analyses reveal an androgen-driven somatic alteration landscape in early-onset prostate cancer. Cancer Cell. 2013;23(2): 159-70.

4. Spratt DE, Zumsteg ZS, Feng FY, Tomlins SA. Translational and clinical implications of the genetic landscape of prostate cancer. Nat Rev Clin Oncol. 2016;13(10):597-610.

5. Cooperberg MR, Carroll PR, Klotz L. Active surveillance for prostate cancer: progress and promise. J Clin Oncol. 2011;29(27):3669-76.

6. Koo KM, Mainwaring PN, Tomlins SA, Trau M. Merging new-age biomarkers and nanodiagnostics for precision prostate cancer management. Nat Rev Urol. 2019:16(5):302-17.

7. Narayan VM. A critical appraisal of biomarkers in prostate cancer. World J Urol. 2019. https://doi.org/10.1007/s00345-019-02759-x.

8. Fraser M, Sabelnykova VY, Yamaguchi TN, Heisler LE, Livingstone J, Huang V, et al. Genomic hallmarks of localized, non-indolent prostate cancer. Nature. 2017:541(7637):359-64.

9. Gerhauser C, Favero F, Risch T, Simon R, Feuerbach L, Assenov Y, et al. Molecular evolution of early-onset prostate cancer identifies molecular risk markers and clinical trajectories. Cancer Cell. 2018;34(6):996-1011.

10. Clinton TN, Bagrodia A, Lotan Y, Margulis V, Raj GV, Woldu SL. Tissue-based biomarkers in prostate cancer. Expert Rev Precis Med Drug Dev. 2017;2(5): 249-60

11. Yegnasubramanian S, De Marzo AM, Nelson WG. Prostate cancer epigenetics: from basic mechanisms to clinical implications. Cold Spring Harb Perspect Med. 2019;9(4). https://doi.org/10.1101/cshperspect.a030445

12. Cancer Genome Atlas Research Network. The molecular taxonomy of primary prostate cancer. Cell. 2015;163(4):1011-25.

13. Yang M, Park JY. DNA methylation in promoter region as biomarkers in prostate cancer. In: Dumitrescu RG, Verma M, editors. Cancer epigenetics: methods and protocols. Totowa: Humana Press; 2012. p. 67-109. https://doi. org/10.1007/978-1-61779-612-8_5.

14. Haldrup C, Mundbjerg K, Vestergaard EM, Lamy P, Wild P, Schulz WA, et al. DNA methylation signatures for prediction of biochemical recurrence after radical prostatectomy of clinically localized prostate cancer. J Clin Oncol. 2013:31(26):3250-8.

15. Camacho DM, Collins KM, Powers RK, Costello JC, Collins JJ. Next-generation machine learning for biological networks. Cell. 2018;173(7):1581-92.

16. Breiman L. Random forests. Mach Learn. 2001:45(1):5-32.

17. Stelloo S, Nevedomskaya E, Kim Y, Schuurman K, Valle-Encinas E, Lobo J, et al. Integrative epigenetic taxonomy of primary prostate cancer. Nat Commun. 2018;9(1):4900

18. Li F, Wan M, Zhang B, Peng Y, Zhou Y, Pi C, et al. Bivalent histone modifications and development. Curr Stem Cell Res Ther. 2018;13(2):83-90.

19. McLean CY, Bristor D, Hiller M, Clarke SL, Schaar BT, Lowe CB, et al. GREAT improves functional interpretation of cis-regulatory regions. Nat Biotechnol. 2010;28(5):495-501.

20. Hastie T, Tibshirani R, Friedman J. The elements of statistical learning: data mining, inference, and prediction. Springer Series in Statistics. 2nd ed. New York City: Springer; 2009.

21. Du Q, Bert SA, Armstrong NJ, Caldon CE, Song JZ, Nair SS, et al. Replication timing and epigenome remodelling are associated with the nature of chromosomal rearrangements in cancer. Nat Commun. 2019;10(1):416.

22. Zhou W, Dinh HQ, Ramjan Z, Weisenberger DJ, Nicolet CM, Shen $\mathrm{H}$, et al. DNA methylation loss in late-replicating domains is linked to mitotic cell division. Nat Genet. 2018:50(4):591-602

23. Jiang C, Wang J, Dong C, Wei W, Li J, Li X. Membranous type matrix metalloproteinase 16 induces human prostate cancer metastasis. Oncol Lett. 2017;14(3):3096-102.
24. Gutierrez ML, Corchete L, Teodosio C, Sarasquete ME, del Mar AM, Iglesias $M$, et al. Identification and characterization of the gene expression profiles for protein coding and non-coding RNAs of pancreatic ductal adenocarcinomas. Oncotarget. 2015:6(22):19070-86.

25. Gong J, Li Y, Liu CJ, Xiang Y, Li C, Ye Y, et al. A pan-cancer analysis of the expression and clinical relevance of small nucleolar RNAs in human cancer. Cell Rep. 2017;21(7):1968-81.

26. Hernandez SJ, Dolivo DM, Dominko T. PRMT8 demonstrates variant-specific expression in cancer cells and correlates with patient survival in breast, ovarian and gastric cancer. Oncol Lett. 2017;13(3):1983-9.

27. Lee J, Sayegh J, Daniel J, Clarke S, Bedford MT. PRMT8, a new membranebound tissue-specific member of the protein arginine methyltransferase family. J Biol Chem. 2005:280(38):32890-6.

28. Cardama GA, Gonzalez N, Maggio J, Menna PL, Gomez DE. Rho GTPases as therapeutic targets in cancer (review). Int J Oncol. 2017:51(4):1025-34.

29. Yeh CR, Da J, Song W, Fazili A, Yeh S. Estrogen receptors in prostate development and cancer. Am J Clin Exp Urol. 2014;2(2):161-8.

30. Noguchi A, Adachi S, Yokota N, Hatta T, Natsume T, Kawahara H. ZFP36L2 is a cell cycle-regulated CCCH protein necessary for DNA lesion-induced Sphase arrest. Biol Open. 2018;7(3):bio031575.

31. Suk FM, Chang CC, Lin RJ, Lin SY, Liu SC, Jau CF, et al. ZFP36L1 and ZFP36L2 inhibit cell proliferation in a cyclin D-dependent and p53independent manner. Sci Rep. 2018:8(1):2742.

32. Baniwal SK, Khalid O, Gabet Y, Shah RR, Purcell DJ, Mav D, et al. Runx2 transcriptome of prostate cancer cells: insights into invasiveness and bone metastasis. Mol Cancer. 2010;9:258.

33. Hoogland AM, Bottcher R, Verhoef E, Jenster G, van Leenders GJ. Geneexpression analysis of Gleason grade 3 tumor glands embedded in lowand high-risk prostate cancer. Oncotarget. 2016;7(25):37846-56.

34. Prensner JR, Rubin MA, Wei JT, Chinnaiyan AM. Beyond PSA: the next generation of prostate cancer biomarkers. Sci Transl Med. 2012;4(127): $127 \mathrm{rv3}$

35. Claus R, Lucas DM, Stilgenbauer S, Ruppert AS, Yu L, Zucknick M, et al. Quantitative DNA methylation analysis identifies a single CpG dinucleotide important for ZAP-70 expression and predictive of prognosis in chronic lymphocytic leukemia. J Clin Oncol. 2012;30(20):2483-91.

36. Litovkin K, Van Eynde A, Joniau S, Lerut E, Laenen A, Gevaert T, et al. DNA methylation-guided prediction of clinical failure in high-risk prostate cancer. PLoS One. 2015;10(6):e0130651.

37. Ahmad AS, Vasiljevic N, Carter P, Berney DM, Moller H, Foster CS, et al. A novel DNA methylation score accurately predicts death from prostate cancer in men with low to intermediate clinical risk factors. Oncotarget. 2016;7(44):71833-40.

38. Bhasin JM, Lee BH, Matkin L, Taylor MG, Hu B, Xu Y, et al. Methylome-wide sequencing detects DNA hypermethylation distinguishing indolent from aggressive prostate cancer. Cell Rep. 2015;13(10):2135-46.

39. Geybels MS, Wright JL, Bibikova M, Klotzle B, Fan JB, Zhao S, et al. Epigenetic signature of Gleason score and prostate cancer recurrence after radical prostatectomy. Clin Epigenetics. 2016:8:97.

40. Stirzaker C, Taberlay PC, Statham AL, Clark SJ. Mining cancer methylomes: prospects and challenges. Trends Genet. 2014;30(2):75-84.

41. Mundbjerg K, Chopra S, Alemozaffar M, Duymich C, Lakshminarasimhan R, Nichols PW, et al. Identifying aggressive prostate cancer foci using a DNA methylation classifier. Genome Biol. 2017;18(1):3.

42. Wei L, Wang J, Lampert E, Schlanger S, DePriest AD, Hu Q, et al. Intratumoral and intertumoral genomic heterogeneity of multifocal localized prostate cancer impacts molecular classifications and genomic prognosticators. Eur Urol. 2017;71(2):183-92.

43. Brinkman AB, Nik-Zainal S, Simmer F, Rodriguez-Gonzalez FG, Smid M, Alexandrov LB, et al. Partially methylated domains are hypervariable in breast cancer and fuel widespread CpG island hypermethylation. Nat Commun. 2019:10(1):1749.

44. Sinha A, Huang V, Livingstone J, Wang J, Fox NS, Kurganovs N, et al. The proteogenomic landscape of curable prostate cancer. Cancer Cell. 2019: 35(3):414-27.

45. Ali RG, Bellchambers HM, Arkell RM. Zinc fingers of the cerebellum (Zic): transcription factors and co-factors. Int J Biochem Cell Biol. 2012;44(11): 2065-8.

46. Ishiguro A, Hatayama M, Otsuka Ml, Aruga J. Link between the causative genes of holoprosencephaly: Zic2 directly regulates Tgif1 expression. Sci Rep. 2018;8(1):2140 
47. Marchini S, Poynor E, Barakat RR, Clivio L, Cinquini M, Fruscio R, et al. The zinc finger gene ZIC2 has features of an oncogene and its overexpression correlates strongly with the clinical course of epithelial ovarian cancer. Clin Cancer Res. 2012;18(16):4313-24.

48. Lu SX, Zhang CZ, Luo RZ, Wang CH, Liu LL, Fu J, et al. Zic2 promotes tumor growth and metastasis via PAK4 in hepatocellular carcinoma. Cancer Lett. 2017; $402: 71-80$.

49. Inaguma $\mathrm{S}$, Ito $H$, Riku M, Ikeda $H$, Kasai K. Addiction of pancreatic cancer cells to zinc-finger transcription factor ZIC2. Oncotarget. 2015;6(29):28257-68.

50. Egevad L, Ahmad AS, Algaba F, Berney DM, Boccon-Gibod L, Comperat E, et al. Standardization of Gleason grading among 337 European pathologists. Histopathology. 2013;62(2):247-56.

51. Humphrey PA, Moch H, Cubilla AL, Ulbright TM, Reuter VE. The 2016 WHO classification of tumours of the urinary system and male genital organs-part B: prostate and bladder tumours. Eur Urol. 2016;70(1):106-19.

52. Assenov Y, Muller F, Lutsik P, Walter J, Lengauer T, Bock C. Comprehensive analysis of DNA methylation data with RnBeads. Nat Methods. 2014;11(11):1138-40.

53. Brocks D, Assenov Y, Minner S, Bogatyrova O, Simon R, Koop C, et al. Intratumor DNA methylation heterogeneity reflects clonal evolution in aggressive prostate cancer. Cell Rep. 2014;8(3):798-806.

54. Ritchie ME, Phipson B, Wu D, Hu Y, Law CW, Shi W, et al. limma powers differential expression analyses for RNA-sequencing and microarray studies. Nucleic Acids Res. 2015;43(7):e47.

55. Pageaud Y, Plass C, Assenov Y. Enrichment analysis with EpiAnnotator. Bioinformatics. 2018;34(10):1781-3.

56. Sing T, Sander O, Beerenwinkel N, Lengauer T. ROCR: visualizing classifier performance in R. Bioinformatics. 2005;21(20):3940-1.

57. Schlomm T, Iwers L, Kirstein P, Jessen B, Kollermann J, Minner S, et al. Clinical significance of p53 alterations in surgically treated prostate cancers. Mod Pathol. 2008;21(11):1371-8.

58. Kononen J, Bubendorf L, Kallioniemi A, Barlund M, Schraml P, Leighton S, et al. Tissue microarrays for high-throughput molecular profiling of tumor specimens. Nat Med. 1998;4(7):844-7.

59. Minner S, Enodien M, Sirma H, Luebke AM, Krohn A, Mayer PS, et al. ERG status is unrelated to PSA recurrence in radically operated prostate cancer in the absence of antihormonal therapy. Clin Cancer Res. 2011;17(18):587888.

60. Burkhardt L, Fuchs S, Krohn A, Masser S, Mader M, Kluth M, et al. CHD1 is a 5 q21 tumor suppressor required for ERG rearrangement in prostate cancer. Cancer Res. 2013;73(9):2795-805.

61. Kluth M, Hesse J, Heinl A, Krohn A, Steurer S, Sirma H, et al. Genomic deletion of MAP 3K7 at $6 q 12-22$ is associated with early PSA recurrence in prostate cancer and absence of TMPRSS2:ERG fusions. Mod Pathol. 2013; 26(7):975-83.

62. Krohn A, Diedler T, Burkhardt L, Mayer PS, De Silva C, Meyer-Kornblum M, et al. Genomic deletion of PTEN is associated with tumor progression and early PSA recurrence in ERG fusion-positive and fusion-negative prostate cancer. Am J Pathol. 2012;181(2):401-12.

63. Krohn A, Seidel A, Burkhardt L, Bachmann F, Mader M, Grupp K, et al. Recurrent deletion of 3p13 targets multiple tumour suppressor genes and defines a distinct subgroup of aggressive ERG fusion-positive prostate cancers. J Pathol. 2013;231(1):130-41.

\section{Publisher's Note}

Springer Nature remains neutral with regard to jurisdictional claims in published maps and institutional affiliations.

Ready to submit your research? Choose BMC and benefit from:

- fast, convenient online submission

- thorough peer review by experienced researchers in your field

- rapid publication on acceptance

- support for research data, including large and complex data types

- gold Open Access which fosters wider collaboration and increased citations

- maximum visibility for your research: over $100 \mathrm{M}$ website views per year

At BMC, research is always in progress.

Learn more biomedcentral.com/submissions 